\title{
The Existence of Stable Electron Trajectories in the Atom Proved by the Ether Theory
}

\author{
David Zareski ${ }^{1,2}$ \\ ${ }^{1}$ I.A.I. Israel Aerospace Industries, Yehud, Israel \\ ${ }^{2}$ former from Université Paul Sabatier, Toulouse, France \\ Correspondence: David Zareski, I.A.I. Israel Aerospace Industries, Yehud, Israel. E-mail: zareski@inter.net.il \\ Received: January 4, 2018 \\ Accepted: January 23, 2018 \\ Online Published: January 30, 2018 \\ doi:10.5539/apr.v10n1p48 \\ URL: https://doi.org/10.5539/apr.v10n1p48
}

\begin{abstract}
The electron on a trajectory around a nucleus radiates energy since it is accelerated and, following the classical theories of physics, should fall on the nucleus. But since it does not fall, it exists a cause, not taken into account by these theories, that prevents it from this fall. Indeed, in our precedent publications we showed that the fields and the particles are changes in a specific elastic medium called "ether". In particular, that a particle is a globule called "single-particle wave" that vibrates in the ether and is denoted $\hat{\xi}$. This $\widehat{\xi}$ that moves at the velocity $\boldsymbol{V}$ of the particle, contains all the parameters relative to it, and modulates the amplitude of the superposition of a wave called "phase-wave", denoted $\boldsymbol{\xi}$, (and not $\widehat{\xi}$ ) of phase velocity $\boldsymbol{V}_{\boldsymbol{p}}$. In the present paper, one considers that the electron moves on a circle of circumference $C$, under the attraction of a nucleus. $\xi$ is then present simultaneously several times, e.g., $\mathrm{N}$ times, at each point of this circle, that is, in $\widehat{\xi}$, which is influenced by these $N$ superpositions, due to the fact that $\xi$ interferes with itself at each round. It appears then the two possible cases: the resonant and the nonresonant.

In the resonant case, $C$ contains an integer number of wave lengths $\lambda$ of $\xi$ that therefore superposes itself at each round in a constructive addition, and creates a relatively large $\widehat{\xi}$, i.e., of a relatively large energy. The electron that radiates then only a limited percentage of its large energy, does not fall on the nucleus.

In the nonresonant case, $C$ contains a non-integer number of $\lambda$, then $\xi$ superposes itself at each round in a destructive addition, and creates a much smaller $\widehat{\xi}$, i.e., of much smaller energy. The electron that radiates then almost the same energy as in the resonant case, loses in fact a much larger percentage of its energy than in the resonant case, cannot remain in this state.
\end{abstract}

\section{Résumé}

L'électron sur une trajectoire autour d'un noyau d'atome radie de l'énergie puisqu'il est accéléré et, suivant les théories classiques de la physique, devrait tomber sur ce noyau. Mais puisqu'il n'y tombe pas, il existe une cause, non prise en ligne de compte par ces théories qui l'empêche d'y tomber. En effet, dans nos précédentes publications nous avons prouvé que les champs et les particules sont des modifications dans le milieu élastique dénommé "éther". En particulier, qu'une particule est un globule dans l'éther. Ce globule dénommé "single-particle wave" et dénoté $\widehat{\xi}$, se déplace à la vitesse $\boldsymbol{V}$ de la particule, contient tous les paramètres relatifs a elle, module l'amplitude d'une éventuelle superposition d'une onde dénommée «onde de phase» et dénotée $\xi$, de vitesse de phase $\boldsymbol{V}_{\boldsymbol{p}}$, reliée à $V$. Dans le présent article nous considérons que l'électron se meut sur un cercle de circonférence $C$ et est soumis à l'attraction du noyau. $\xi$ est donc présente simultanément plusieurs fois, e.g., $\mathrm{N}$ fois, a chaque point de ce cercle, c'est à dire dans $\widehat{\xi}$, qui est influence par ces $\mathrm{N}$ superpositions, dues au fait que $\xi$ interfère avec elle-même à chaque tour, il apparait alors deux cas possibles : le resonnant et le nonresonnant.

Dans le cas résonant, $C$ contient un nombre entier de longueurs d'onde $\lambda$ de $\xi$ qui donc se superposent elles même à chaque tour en une addition constructive, et créent une relativement grande $\widehat{\xi}$, i.e., de relativement grande énergie. L'électron qui radie alors seulement un percentage limité de cette grande énergie ne tombe pas sur le noyau.

Dans le cas nonrésonant, $C$ contient un nombre non-entier de $\lambda$, alors $\xi$ se superpose à chaque tour en une addition destructrice, et crée un beaucoup plus petit $\widehat{\xi}$, i.e., de beaucoup plus petite énergie. L'électron qui radie 
alors presque la même énergie que dans le cas résonant, perd en fait un beaucoup plus grand percentage de son énergie que dans le cas résonant ne peut pas rester dans cet état.

Keywords: Ether elasticity theory; phase-waves; single-particle waves; resonant and no-resonant superpositions of waves in an atom; conditions for an atom electron to not fall in a lower state of energy

\section{Introduction, Recalls, and Notations}

One denotes $\hat{C}(r)$ a circular trajectory of radius $r$, around the nucleus of an atom. Following the theories of physics that do not take into account the existence of the elastic medium called "ether", it should not exist any stable state of an electron describing a $\hat{C}(r)$, since following these theories, such an accelerated electron should emit energy and therefore finally fall on the nucleus. But if it was so, it would not exit any atom and therefore any molecule, and therefore any life in the universe. Now, since there are electrons of atoms that do not fall on the nucleus despite the existence of attractive forces and the loss of electromagnetic energy due to the electron acceleration, and furthermore, since the mechanical laws are generally valid, it follows that:

"it must exist a physical phenomenon, supplementary to those that we now, that in particular, prevents the electron of an atom from falling on the nucleus".

Indeed, as mentioned here above, shown in our precedent publications and recalled here below, this physical phenomenon is the existence of the elastic medium called "ether", governed in the case treated here by the equation of elasticity (4.5) of Zareski (2015) and also by the continuity equation (1.3) (ibid), of which local changes are the fields and the particles. Before entering in the heart of the subject, we recall now some properties and results regarding the ether demonstrated in our previous publications.

In the ether are propagated waves $\xi$, called "phase-waves", of phase velocity $\boldsymbol{V}_{P}$, of which a part is generally enclosed in a globule in the ether. Such a globule denoted $\widehat{\xi}$, is called "single-particle wave", is of finite volume, moves at the velocity $\boldsymbol{V}$ of the particle, and contains all the parameters relative this particle, Cf. Zareski (2001, 2012, 2013a). (Remark: do not confound $\xi$ and $\hat{\xi})$.

We recall now some properties of $\xi$ and $\hat{\xi}$. When the mass of this particle is zero, then $\hat{\xi}$ is a photon and $\xi$ yields an electromagnetic field. When the massive particle is immobile, then Cf. Sec. IX of Zareski (2015), $\hat{\xi}$ creates a static field and if the volume of this particle is sufficiently small, it is a "black hole". Furthermore, we demonstrated, Cf. Zareski (2014), and Sec. VIII of Zareski (2015), that the electron spin is the velocity of the points of the ether near to the moving $\hat{\xi}$; that, Cf. Zareski (2013b), and Sec. VI of Zareski (2015), the strong nuclear interaction is due to elastic and general relativistic effects on massive and electrically charged particles even of same sign when they are sufficiently close one to the other; that, Cf. Zareski (2014b), and Sec. VII of Zareski (2015), the Schrödinger equation is a particular form of the equations that govern the ether; and that, Cf. Zareski (2011, 2014a), and Sec. IX of Zareski (2015), electromagnetism which is a particular case of the elasticity theory, is also the Newton approximation of Einstein's general relativity. Now, the expression for the phase-wave $\xi$ is, Cf. Sec. IV. 2 of Zareski (2015),

$$
\xi=\xi_{0} \sin \left[\omega\left(t-\int d \ell / V_{P}\right)\right]
$$

In Eq. (1), t denotes the time, $\omega$, the pulsation related to the frequency $v$ by the relation $\omega=2 \pi v$, and $d \ell$, the element of trajectory. Denoting

$$
\operatorname{SINC}(x) \equiv \sin (x) / x,
$$

the expression for the single-particle wave $\hat{\xi}$ is, Cf. Eq. (5.7), (ibid),

$$
\widehat{\xi}=\xi \frac{1}{\Delta \omega} \int_{-\Delta \omega / 2}^{\Delta \omega / 2} \exp \left[i\left(t-\int d \ell / V\right) \vartheta\right] d \vartheta
$$

that is,

$$
\widehat{\xi}=\xi \operatorname{SINC}\left[(\Delta \omega / 2)\left(t-\int d \ell / V\right)\right],
$$

where, Cf. Sec. III. 6, (ibid), $V_{P}$ and $V$ are connected by the relation

$$
\frac{\partial}{\partial E_{T}}\left(\frac{E_{T}}{V_{P}}\right)=\frac{1}{V} .
$$

\section{Generalities on the Phase-Wave and on the Single-Particle Wave Associated to the Electron Describing a Circle Around an Atom Nucleus}

One considers that during a time interval defined in the continuation, the electron moves on a $\hat{C}(r)$. The phasewave is then present simultaneously several times, e.g., $\mathrm{N}$ times, at each point of $\hat{C}(r)$, that is, also in the globule 
$\widehat{\xi}$, which therefore is influenced by these $N$ superpositions, due to the fact that the phase-wave interferes with itself at each round. Furthermore, one considers that the phase-wave of an electron on $\hat{C}(r)$, is of constant amplitude $\xi_{0}$, and propagated with the phase velocity $V_{P}$ of constant amplitude on this $\hat{C}(r)$ under the attraction of the nucleus of, e.g., a hydrogen atom, that is,

$$
\int d \ell / V_{P}=r / V_{P} \int d \theta=\theta r / V_{P}+\theta_{0} r / V_{P},
$$

where $\theta$ denotes the running angle on this circle such that $\ell=r \theta$, and $\theta_{0}$ an arbitrary constant angle. To the purpose of this paper, one takes $\theta_{0}=2 \pi k$, where $k$ is an integer, (4) becomes then

$$
\int d \ell / V_{P}=\theta r / V_{P}+2 \pi k r / V_{P} .
$$

In particular the expression for $V_{P}$ is, Cf., e.g., Eqs. (3.56) and (3.63) of Zareski (2015),

$$
V_{P}=\frac{c \gamma E_{T}}{\left(E_{T}+e A_{4}\right) B}
$$

where $B$ is defined by

$$
B \equiv \sqrt{1-\left(\frac{\gamma m c^{2}}{E_{T}+e A_{4}}\right)^{2}} .
$$

In these expressions for $V_{P}$ and,$E_{T}$ denotes the electron total energy, $\gamma$, the Schwarzschild element defined in, e.g., Sec. III. 8 of Zareski (2015), $e$, the electric charge of the electron, $A_{4}$, the electromagnetic potential to which is submitted the electron, and $m$, the electron mass.

Now, one denotes $n_{0}$, a positive integer, and " $a$ ", a null or positive number smaller than 1 , such that when the electron moves on $\hat{C}(r)$ then $C$ will contain $n_{0}+a$ wave lengths $\lambda$, that is:

$$
r \omega / V_{P} \equiv 2 \pi r / \lambda=n_{0}+a .
$$

Therefore, the term $\omega \int d \ell / V_{P}$ that appears in Eq. (1) becomes taking Eq. (5) and (7) into account:

$$
\omega \int d \ell / V_{P}=(\theta+2 k \pi)\left(n_{0}+a\right) .
$$

In this case where the electron describes $\hat{C}(r)$, the phase-wave associated to this electron which theoretically is of large length Cf. Eq. (1), wraps around the nucleus. Therefore, if $N$ denotes the number of times that the phase-wave wraps around the nucleus, then $N$ can be very large. The expression for the phase-wave denoted $\xi\left(N, n_{0}+a\right)$ in this case instead of , is:

$$
\xi\left(N, n_{0}+a\right)=\xi_{0} \Sigma_{k=0}^{k=N} \sin \left[\omega t-(\theta+2 k \pi)\left(n_{0}+a\right)\right],
$$

that is

$$
\xi\left(N, n_{0}+a\right)=\xi_{0} I\left\{\exp \left[i\left[\omega t-\theta\left(n_{0}+a\right)\right]\right] \cdot \Sigma_{k=0}^{k=N} \exp (-i 2 k \pi a)\right\} .
$$

where $I\{\}$ indicates the "imaginary part of \{\} ". We define now the single-particle wave $\widehat{\xi}$ denoted more precisely in this case $\widehat{\xi}\left(N, n_{0}+a\right)$. To this end one denotes

$$
\tau(a) \equiv t-\left(n_{0}+a\right) \theta \lambda /(2 \pi V)
$$

where the expression for the particle velocity $V$ is, Cf. e.g., Eq. (3.55) of Zareski (2015),

$$
V=c \gamma B \text {, }
$$

Eq. (9) becomes then:

$$
\hat{\xi}\left(N, n_{0}+a\right)=\xi\left(N, n_{0}+a\right) \operatorname{SINC}[(\Delta \omega / 2) \tau(a)] .
$$

\section{The Single-Particle Wave Associated to the Electron Describing a Circle Around an Immobile Atom Nucleus in the Two Cases: The Resonant and the Nonresonant}

\subsection{The Resonant Electron Phase -Wave and Single-Particle Wave}

\subsubsection{The Resonant Electron Phase-Wave}

This resonant case is the one where $a=0$. Indeed, in this case, Eq. (9) becomes

$$
\xi\left(N, n_{0}\right)=(1+N) \xi_{0} \sin \left(\omega t-\theta n_{0}\right) .
$$

and in particular for $\theta=\theta_{1}$, such that $\sin \left(\omega t-\theta_{1} n_{0}\right)=1$ that is, for

where $n$ is an arbitrary integer, then (13) becomes

$$
\theta_{1}=[\omega t-(2 n \pi+\pi / 2)] / n_{0} \text {, }
$$

$$
\xi\left(N, n_{0}\right)=(1+N) \xi_{0}
$$




\subsubsection{The Resonant Electron Single-Particle Wave}

We have proven here above by the use of the ether elasticity theory that the electron is a superposition of the phasewave in a globule as it appears generally in Eq. (12). Now, the resonant case is defined by $=0$, in this case

Eq. (12) becomes

$$
\widehat{\xi}\left(N, n_{0}\right)=(1+N) \xi_{0} \sin \left(\omega t-\theta n_{0}\right) \operatorname{SINC}[(\Delta \omega / 2) \tau(0)] .
$$

\subsection{The Nonresonant Electron Phase -Wave and Single-Particle Wave}

\subsubsection{The Nonresonant Electron Phase-Wave}

This nonresonant case is the one where strictly $0<a<1$. In this case, the explicit expression for the term $\Sigma_{k=0}^{k=N} \exp (-i 2 k \pi a)$ that appears in (9) is

$$
\Sigma_{k=0}^{k=N} \exp (-i 2 k \pi a)=\left[1-e^{-i 2 \pi(N+1) a}\right] /\left(1-e^{-i 2 \pi a}\right) .
$$

It appears, for example, that for $a=0.5$, then Eq. (17) becomes

i.e., Eq. (9) becomes

$$
\Sigma_{k=0}^{k=N} \exp (-i k \pi)=0.5\left[1-(-1)^{N+1}\right],
$$

One sees that

$$
\xi\left(N, n_{0}+0.5\right)=0.5\left[1-(-1)^{N+1}\right] \xi_{0} \sin \left[\omega t-\theta\left(n_{0}+0.5\right)\right]
$$

$$
\left|\xi\left(N, n_{0}+0.5\right)\right| \leq \xi_{0}
$$

which is much smaller than $(1+N) \xi_{0}$ as it appears in the case where $a=0$, i.e., in (15).

\subsubsection{The Nonresonant Electron Single-Particle Wave}

We have proven here above by the use of the ether elasticity theory that the electron is a superposition of the phasewave in globule as it appears generally in Eq. (12). Now, the nonresonant case is defined by $\in] 0,1[$. In the particular case where $a=0.5$, Eq. (12) becomes

$$
\hat{\xi}\left(N, n_{0}+0.5\right)=0.5\left[1-(-1)^{N+1}\right] \xi_{0} \sin \left[\omega t-\theta\left(n_{0}+0.5\right)\right] \operatorname{SINC}[(\Delta \omega / 2) \tau(0.5)],
$$

which is much smaller than $\hat{\xi}\left(N, n_{0}\right)$ defined in Eq. (16).

\section{Conclusion}

As it appears from the relations (21) and (16): for $a=0$, i.e., in the resonant state, the electron energy is sensibly $N^{3}$ times larger than in the no-resonant state defined, e.g., for $a=1 / 2$. This fact implies that even if the electron emits energy due to its accelerated motion around the nucleus, then when

$a=0$, it remains sufficiently energy since $\boldsymbol{N}$ can be very large, to prevent the electron from falling quickly in a lower empty state of the atom energy, and also prevent it completely from falling on a occupied lower state of energy. While in the case where for example $a=1 / 2$, it does not remain enough energy to the electron to continue to be in this state of energy.

The important point is that: for $a=0$, i.e., in a resonant state, then, to the electron in the lowest quantum state of energy of, e.g., a hydrogen atom, it remains sufficiently energy to prevent it from falling on the nucleus. At my knowledge, only the take into account of the ether theory permits to prove this fact.

This paper is based on the hypothesis of the existence of an elastic medium called "ether" preconized by Maxwell and Einstein, and demonstrated in my previous publications following with, the fields and the particles are changes in the ether.

The existence of the ether can be proven by the fact that the light velocity does not depend upon the emitter velocity as preconized by Einstein.

Furthermore, the fact that the electron in the resonant state of an atom loses energy but not enough to fall on the nucleus, is of the same form as a resonance in a laser.

\section{References}

Zareski, D. (2001). The elastic interpretation of electrodynamics. Foundations of Physics Letters, 14(5), 447-469.

Zareski, D. (2012). On the elasto-undulatory interpretation of fields and particles. Physics Essays, 25(2).

Zareski, D. (2013). Fields and wave-particle reciprocity as changes in an elastic medium: The ether. Physics Essays, 26(2), 288-295. 
Zareski, D. (2013). The elasto-gravitational interpretation of the "strong interaction". Physics Essays, 26(4), 548552.

Zareski, D. (2014). The Electron Spin as Resulting From the Ether Elasticity. Applied Physics Research, 6(5), 41.

Zareski, D. (2014). The ether theory as implying that electromagnetism is the Newtonian approximation of general relativity. Physics Essays, 27(4), 517-522.

Zareski, D. (2014). The Quantum Mechanics as Also a Case of the Ether Elasticity Theory. Applied Physics Research, 6(4), 48.

Zareski, D. (2015). Unification of Physics by the Ether Elasticity Theory. LAP LAMBERT Academic Publishing.

\section{Copyrights}

Copyright for this article is retained by the author(s), with first publication rights granted to the journal.

This is an open-access article distributed under the terms and conditions of the Creative Commons Attribution license (http://creativecommons.org/licenses/by/4.0/). 